\title{
[Souvenirs sur Auguste Chevalier]
}

Un manuscrit inédit

\section{Roland Portères}

\section{(2) OpenEdition \\ Journals}

Édition électronique

URL : http://journals.openedition.org/ethnoecologie/5802

DOI : $10.4000 /$ ethnoecologie.5802

ISSN : 2267-2419

Éditeur

Laboratoire Eco-anthropologie et Ethnobiologie

\section{Référence électronique}

Roland Portères, «[Souvenirs sur Auguste Chevalier] », Revue d'ethnoécologie [En ligne], 16 | 2019, mis en ligne le 31 décembre 2019, consulté le 06 janvier 2020. URL : http://journals.openedition.org/ ethnoecologie/5802; DOI : 10.4000/ethnoecologie.5802

Ce document a été généré automatiquement le 6 janvier 2020

\section{(c) $($ ) $\odot$ (S)}

Revue d'ethnoécologie est mis à disposition selon les termes de la licence Creative Commons Attribution - Pas d'Utilisation Commerciale - Pas de Modification 4.0 International. 


\title{
[Souvenirs sur Auguste Chevalier]
}

\author{
Un manuscrit inédit
}

\author{
Roland Portères
}

\section{NOTE DE L'ÉDITEUR}

Dans les archives du laboratoire d'ethnobotanique, Catherine Hoare a retrouvé un manuscrit inédit de Roland Portères sur Auguste Chevalier, qu'elle a retranscrit. Il n'était pas daté et fut probablement écrit à la fin de sa vie, et rien ne permet de reconstituer la finalité de ce texte de souvenirs personnels.

Nous publions ici, augmenté de notes complémentaires et bibliographiques, ce texte riche et révélateur des deux personnalités fondatrices de l'ethnobotanique du Muséum. Les parties transcrites en italiques étaient soulignées par Portères dans son manuscrit. Nous avons également respecté les nombreuses majuscules et l'orthographe.

Auguste Chevalier (1873-1956) avait été titulaire de la chaire de Productions coloniales d'origine végétale au Muséum national d'Histoire naturelle en 1929, en transformation d'un laboratoire d'agronomie coloniale de l'EPHE et du MNHN, fondé en 1911. Il a été un des botanistes découvreurs de la flore d'Afrique occidentale et d'Afrique centrale à la fin du XIXe et au début du XXe siècle, et a inventorié et documenté les plantes utiles des régions tropicales, ainsi que les procédés d'agriculture locale. Il avait fondé la Revue de botanique appliquée en 1921, dont notre revue est héritière, et que l'on peut consulter en ligne (http://www.persee.fr/web/revues/home/prescript/revue/jatba). On trouvera des informations détaillées sur la vie et l'œuvre de ce précurseur de l'ethnobotanique dans Leroy (1957) et dans Bonneuil (1996).

Roland Portères (1906-1974) lui a succédé en 1948, et il transforma ensuite la chaire (devenue Agronomie tropicale), en chaire (et laboratoire) d'Ethnobotanique en 1963. Agronome spécialiste des céréales africaines, il a donné une impulsion décisive pour le développement des recherches en ethnobotanique et plus largement en ethnoécologie, bien au-delà de l'Afrique tropicale. Pour publier ces travaux, il avait fondé en 1954 le Journal d'agriculture traditionnelle et de botanique appliquée, qui a succédé à la RBA. Sur la vie et l'œuvre de Portères, on consultera Leroy (1974). 
On trouvera des informations plus détaillées sur ces savants et leur influence dans Bahuche \& Lizet (2003) sur l'ethnobotanique au Muséum, et dans notre Revue d'ethnoécologie (Bahuchet 2012 et Hoare 2012 sur les périodiques, Bahuchet et al. 2019 sur les missions tropicales et les collections).

1 En 1926, sortant de l'Institut National d'Agronomie Tropicale et en attendant mon temps de service militaire, je rendis visite au Dr. Auguste Chevalier dont je connaissais plus ou moins son œuvre de Botaniste-Explorateur et surtout par la Revue de Botanique appliquée et d'Agriculture coloniale qui en était déjà à sa $7^{\mathrm{ème}}$ année grâce à André Kopp ${ }^{1}$, Ingénieur Agronome qui avait travaillé auparavant dans les sucreries et plantations de canne de Réunion, Maurice et surtout Guadeloupe/Martinique.

2 À cette époque, le Laboratoire d'Agronomie Coloniale était une dépendance de l'École Pratique des Hautes Études hébergée par le Muséum National d'Histoire Naturelle depuis 1912.

Auparavant avait existé au Jardin des Plantes un laboratoire Colonial, Rue Buffon.

Quand je fus introduit dans le Bureau du Dr. Auguste Chevalier je me sentis intimidé devant cet homme à grosse tête, à cou de taureau, à binocles qui me scrutaient et dont la voix brutale, un peu rauque, s'efforçait d'être douce.

5 Je lui expliquai que je serais désireux pour quelques mois de travailler bénévolement dans son laboratoire, m'étant orienté vers l'Agronomie des Pays chauds et donnant références.

6 Ayant eu des démêlés au début du siècle avec le Jardin Colonial de Nogent-sur-Marne qui assura aussitôt l'éducation de jeunes gens en vue de fournir des cadres aux administrations gouvernementales, aux Sociétés d'Exploitation Agricole en Pays Chauds, il ne me fit pas compliment de ce que j'étais passé par cet Institut d'Agronomie Tropicale : on m'avait mal orienté et c'était grand dommage.

7 Il faut dire qu'il avait eu maille à partir avec Émile Prudhomme ${ }^{2}$, fondateur du Jardin Colonial et ancien Directeur de l'Agriculture à Madagascar, avec Charles Chalot ${ }^{3}$ ancien Directeur du Jardin Botanique de Libreville (île-aux-perroquets), avec Paul Ammann Agronome-Chimiste s'occupant (?) d'analyses concernant plantes et produits de culture ou de cueillette avec Jean Dybowsky ${ }^{4}$, Agronome et Explorateur (à la recherche de la Mission Gentil). Je passe sur le menu fretin, assez nombreux d'ailleurs. [avec le Forestier Commandant Bertin qui prospecta beaucoup sur place les Bois Coloniaux et justement dans les mêmes régions africaines dont s'était occupé Aug. Chevalier]. Toute son acrimonie tournait autour de quelques points principaux :

8 On ne lui avait pas fait place dans l'organisation de Nogent bien qu'il ait tenté d'ouvrir la porte en collaborant au Journal d'Agriculture Tropicale ${ }^{5}$

9 La priorité de découvertes ou d'études concernant des espèces cultivées ou leurs espèces congénères (Coffea excelsa-Dybowski ${ }^{6}$, Riz vivace de l'Afrique, etc.)

10 Il avait engagé une série de publications qui restent toujours du plus grand intérêt dans sa collection (Kolatiers et Kola, Palmier à Huile, Arganier et Karité, Cacaoyers et cacao, Bois de Côte d'Ivoire, du Gabon et du Tonkin, Horticulture coloniale, etc.... Or, à Nogent on éditait aussi sur les Plantes tropicales de culture, l'Agriculture coloniale, le Vanillier et la Vanille, les Fruits tropicaux, etc. et un périodique L'Agronomie Coloniale, puis L'Agronomie Tropicale, issus du Bulletin du Jardin Colonial. 
11 Aug. Chevalier considérait qu'il était le seul en France à être habilité pour traiter de tout ce monde végétal exploité par les Hommes dans les Pays chauds. (enquête bibliographique pensée et exhaustive, concision, conclusions à tirer, protocoles expérimentaux). Là-dessus il retint un travail sur la fermentation du cacao et ses conséquences en industrie chocolatière, d'autant que depuis longtemps il existait un fossé entre chocolatiers britanniques et chocolatiers français, allemands, etc.... Il a publié ce travail dans la Revue de Botanique appliquée et d'Agriculture coloniale (Portères 1927).

19 Malgré les rebuffades et admonestations qu'il distribuait à tous dans son laboratoire, j'étais en quelque sorte agréé.

20 Après de courtes occupations militaires terminées dans les "Opérations de police " (Paul Painlevé ${ }^{11}$ ) de la Guerre du Rif, je revins voir Aug. Chevalier et je débutai à Sorbonne - Science en Botanique et Physiologie.

21 Il avait de très solides et lointaines amitiés avec différents Gouverneurs Généraux (Merlaud-Ponty..., Albert Sarraut ${ }^{12}$, etc....) et des Directeurs au Ministère des Colonies (Gaston Joseph ${ }^{13}$, François..., etc....). Comme il défendait, comme tout le monde à l'époque, la culture du Caféier d'Arabie dans nos dépendances d'Outre-Mer, il avait obtenu que l'on crée en Côte d'Ivoire, à Man, une Station agricole concernant plus particulièrement cette plante, cultivable au-dessus de $800 \mathrm{~m}$ d'altitude dans le Massif des Dans et qu'il connaissait bien (1905).

Il me fit désigner pour un stage préparatoire d'une année à son laboratoire, à Nogent (Quand même !) et sur la place commerciale du Havre qui se refusait dans l'ensemble à cette époque à traiter d'autres cafés que ceux du type Arabica. 

rôle qu'il avait joué antérieurement et compte-tenu qu'il subsistait encore en 1928-1929 beaucoup de dirigeants administratifs et politiques, de commerçants et de particuliers qui l'avaient connu avant 1914, il se faisait " reconnaître » partout (Dakar, Saint-Louis). Il se constituait (ou se reconstituait) « une cour». C'était chez lui un besoin, une nécessité. n'étant pas tellement reconnu des Botanistes et des Agronomes fondamentaux ou d'application ; tout a changé depuis et il fait maintenant figure de grand pionnier), il recréait autour de lui un cercle avec des éléments humains et des faits remontant avec ses 52 années d'âge dont 30 remontant à ses débuts africains, à vingt années en arrière.

C'est à partir de ce moment que nos relations s'établirent toujours avec admonestations, rebuffades, critiques, généreusement distribuées à tous.

À la fin de mon stage il me demanda que je lui sois adjoint pour une mission d'études sur l'Arachide au Sénégal, laquelle dura deux mois (octobre à décembre $1928^{14}$ ). Décidément j'étais sur la bonne piste. contingences sociales et pratiques auxquelles il n'avait jamais pu s'adapter, écrasé par le labeur incroyable qu'il accomplissait (moyenne de 14 heures par jour), mais heureux d'être « visité » par tous, venus de près ou de loin.

littéraires, journalistiques, etc. viennent le voir. Ce sont eux qui s'imposaient, mais pour son mérite, son plaisir et ce sentiment qu'il avait d'être au centre d'un monde ancien, non encore en cours de disparition (Exploration, pionnerisme botanique, découverte de l'Afrique, ressources naturelles saisies depuis longtemps par des «sauvages» et dont il était le prospecteur et l'explicant pour les Sciences et Techniques modernes).

longtemps de Botanique économique, ayant vécu au contact de ceux qui exploitaient des végétaux, il disait son mot, ses mots.

originale intitulée Monographie de l'Arachide (Chevalier 1936) ${ }^{15}$, il s'est montré d'abord à moi : Botaniste de Terrain, Questionneur-Enquêteur, Quémandeur de types variétaux, Historicien d'Agriculture sénégalaise et autre, Fouilleur de documents manuscrits, raviveurs de souvenirs, etc....

Partout où nous allions il se montrait causeur remarquable ouvrant à tous des connaissances remarquables sur ce qui s'était passé avant 1912 dans le domaine africain, avant la terrible guerre de 1914-1918 qui avait tout effacé en cette matière, l'après-guerre ayant apporté nombre d'éléments européens nouveaux et jeunes, peu affairistes et ne s'intéressant pas à un passé récent mais qui leur semblait lointain, historique et relevant de vieux hommes et de vieilles choses, des archives (même fait après 1945). 

toujours bien compris), citant des hommes, les décrivant, mentionnant ce qu'ils avaient fait et leurs erreurs et incompréhensions, en disant du mal et du bien, les excusant en tenant compte des conditions de vie! Tous les grands noms de la colonisation française locale ressortaient avec les œuvres. e tempérament timide, n'offrant jamais aux autres ce qu'il avait de bon en lui-même, ne jugeant jamais devant d'autres sur des questions sociales, sur des groupes humains, il gardait tout. dans les entrailles, dans le cœur. Par la tête, il s'exprimait mal, naïvement, sans considération il faut bien le dire, "négritales". On revoyait en lui Jean-Jacques Rousseau, le « Naturel » pur et bon, incapable de [mal], etc....

souvent remarqué [que] lors de ses «explosions » toujours exprimées avec une voix sourde, lente, voilée, il n'attaquait pas, ne critiquait pas : il constatait. Il était incapable de dire pourquoi il faisait cœur avec l'Homme noir, les Hommes noirs, quels qu'ils soient.

39 Moi qui débarquais sur une terre étrange, j'observais le Bonhomme, le Dr Aug. Chevalier, dans ce qu'il disait et dont je m'imprégnais, dans le Noir tel qu'il le voyait et m'en parlait au cours de nos voyages à deux, de longues journées sur les pistes sableuses, les champs en friche ou presque, les jardins et les mares. Petit à petit, avec lui et à cause de lui, s'est insérée profondément en moi (cela m'a marqué depuis cette époque et m'a même orienté) que l'équilibre Homme-Nature était une réalité historique et sociale dans le cadre d'une autarcie économique traditionnelle non bousculée par une ouverture sur une économie mondiale.

Le champ, la savane à ressources, le bosquet privilégié et protégé, les abords de l'habitat du groupe humain, le jardin et la compostière entourant l'habitation, le statut botanique spécifique et variétal des plantes de grande et de petite culture, le petit et le grand bétail, tout cela était l'Homme.

41 Aug. Chevalier m'a fait comprendre sur le terrain qu'il ne s'agissait pas seulement d'économie de subsistance, d'aménagement et d'exploitation des alentours, d'ouverture sur les économies mondiales, de modes de vie liés à tout cela et à d'autres contraintes (tribales, sociales, administratives, défensives, etc....), de vie matérielle, mais qu'il y avait autre chose, de beaucoup plus grand, de beaucoup plus enveloppant.

Il y avait au travers des champs, des jardins et des végétaux une trame humaine qui faisait que tous ces objets et choses n'étaient rien sans cette dernière; que c'était cette «négritude » d'isolement rural, de pensée et d'action qui donnait existence à tout cela ; qu'il n'était pas question d'une «condition humaine» (Malraux) nécessitée par l'imposition de la Nature et des Hommes, tous contraignants ; que derrière cela, existait le compagnonnage Hommes-Végétaux, au sein d'une conception animiste qui nous 
dépasse présentement (parce que trop métaphysique), que, si condition il y a, c'est la "Condition de la Nature », imposée par l'Homme de plus en plus seul contraignant.

Quand, à deux, nous plongions dans un champ, une friche, un jardin, une rudéralité, Aug. Chevalier m'expliquait, me confrontait avec un monde végétal nouveau, j'avais l'histoire et les pérégrinations d'un végétal ou d'un cortège végétal, les origines humaines de ceux-ci, et, ce faisant, une interprétation du grand voyage déjà accompli par des groupes humains, depuis des siècles, ou par des végétaux depuis des siècles et mêmes des millénaires.

Son " humanisme » était dans cela et à partir de cela. Pauvres agricultures sans moyens instrumentaux, sans connaissances techniques modernes (lesquelles il ignorait à peu près complètement) telle était sans cesse la peinture présentée.

J'ai, à ces moments, eu souvent l'impression qu'il était aussi végétalo-animiste que ceux que nous visitions. Je ne les plaignais jamais, ils étaient dans leur «Condition» (présente) ; mais sans cesse, au cours de nos déplacements, il disait aux autres et à moi qu'il fallait que cela change, que ces gens avaient droit à obtenir mieux dans une nouvelle vie matérielle mondiale et qu'il fallait les aider. C'est d'ailleurs ce qu'il développera dans son ouvrage sur la Monographie de l'Arachide.

J'ai parlé plus haut de sa timidité et des conséquences que cela avait au sein de son laboratoire de Paris. Cette timidité se traduisait aussi, comme je l'ai dit, par une faim de " cour ", un besoin d'honneurs, une reconnaissance de tous, une position sociale même dans des aspects mineurs très communément pratiques mais faisant partie de tout cet ensemble.

47 Un jour, allant par la piste sableuse et malaisée de Bambey à Diourbel ${ }^{16}$, à l'aide d'une vieille Ford haute sur pattes et fille de la Ford de guerre type 1918, nous avancions lentement (chaleur, pneus usés, conducteur somnolent). Aug. Chevalier, qui somnolait aussi plus ou moins, eût un réveil lors d'un passage sur un morceau semi-effondré.

«Chauffeur! Ne pourrais-tu aller plus vite? Nous sommes déjà en retard. Tu sais que chez moi, en France, on s'occupe beaucoup de moi et j'ai l'habitude de toujours voyager en première.

- Ah! Missié, il y a vingt kilomètres que tu es en première.

- Que dit-il ?

- Vous parlez de voyage en 1 ère classe et lui, de mécanique automobile.

- Ah! Je n'ai jamais rien compris à tout cela, mais ce qui m'intéresse c'est que l'on aille plus vite. ».

49 Ce qui fut fait, illico, avec pour résultats : deux pneumatiques crevés en cours de route et une bielle coulée à $2 \mathrm{kms}$ de l'arrivée à Diourbel ! Les crevaisons étaient bénéfiques pour moi et calmaient la hargne de Aug. Chevalier, car chaque fois nous avions pu herboriser autour de deux mares sur émergences de cuirasse latéritique.

50 J'ai subi au cours de cette vie en commun pendant deux mois avec Aug. Chevalier, non pas une formation qui ne pouvait être que partielle, mais une transformation; en ce sens que j'ai appris de lui à faire corps et faire cœur avec l'Afrique Noire, avec les Humanités de l'Afrique; les divers modes de négritude de ce continent que l'on disait autrefois « maudit ».

51 Puis, près de deux années plus tard (1931), étant personnellement au cours du même séjour en Afrique, dans la région de Man (Nord-Ouest forestier de la Côte d'Ivoire), Aug. Chevalier qui venait d'effectuer une Mission d'Études au Soudan ${ }^{17}$ et au Niger, aborda la 
Côte d'Ivoire par le Nord et après quelque temps passé en Bas-Pays désira rentrer à Dakar par l'axe routier de cette époque Abidjan-Man-Fouta-Djallon-TambacoundaDakar. Il tînt à suivre cet itinéraire extrêmement pénible à l'époque pour "me voir ». Nous passâmes quelques journées ensemble. Nous herborisâmes et visitâmes quelques villages et leurs cultures. Il n'avait pas changé dans son physique et son comportement. Ses binocles scrutaient toujours, comme à Paris et au Sénégal.

Ce que je n'avais pas tellement compris près de deux années avant c'est que le rabrouement, le commandement (de Paris) que je n'avais pas subi de sa part au Sénégal, n'existait pas non plus en Côte d'Ivoire ; il se montrait conseiller, aimable, explicant, toujours très détendu. J'en profitai pour faire ramasser une cinquantaine d'échantillons d'arbres et d'arbustes et il se pencha avec plaisir dans le travail d'identification ou d'orientation en ce but, ce qui me servit beaucoup.

Quand nous quittâmes Danané, il me parla à peine, me tînt la tête entre ses deux mains, puis une double tape sur les épaules en me disant «A bientôt ! ».

Le temps passa. Je fréquentais encore le laboratoire de Paris en 1932, en 1935, en 1938. Puis, en 1943, je le revis à Dakar, lors du $1^{\text {er }}$ congrès des Anthropologistes de l'OuestAfricain, organisé par Mr Théodore Monod, Directeur de l'Institut d'Afrique Noire ${ }^{18}$.

L'Homme n'était plus le même. Las, brisé, les yeux regardant le lointain, ne causant que peu et sans raconter de bonnes histoires ou sans rappel de son passé, de son temps vécu en Afrique et pour l'Afrique. J'en fus singulièrement ému, peiné, déconcerté. La Guerre n'était pas encore terminée ; il en souffrait, comme tout le monde, et cela, pensais-je, devait l'avoir marqué.

Cela était certes, mais il y avait tout autre chose à laquelle moi, jeune, je n'avais pas pensé. C'est que deux années plus tard, à peu près, jour pour jour, il serait «admis à faire valoir ses droits à la retraite ». Cela le minait d'avance, le tuait. Non pas qu'il ne pourrait continuer à travailler comme auparavant, en toute liberté, hors des contraintes et contingences administratives. Ce n'était pas cette position de liberté hors administration qui le rongeait.

Un soir, dans sa chambre chaude et mal éclairée où le vol des moustiques stridulait à nos oreilles, il m'expliqua qu'ayant édifié une sorte de Temple à la Botanique appliquée aux Pays chauds, ayant par un labeur presque entièrement consacré à l'Afrique d'abord, puis au Monde, depuis 1898, il devait se retirer et céder tout cela à un autre, un « remplaçant ». Il m'expliqua que toute son œuvre dans ce qu'elle avait de continuation nécessaire devait se poursuivre, et dans le même esprit ; qu'il regrettait de ne pas avoir formé personnellement d'élèves pour assurer sa succession; que lui vivant et en fonction, la question ne s'était jamais posée dans son esprit, confiant en sa force, en ses capacités de travail, en sa notoriété. Mais, brutalement, survenait une date limite et, plus grave, tous ceux dont il s'auréolait antérieurement, ou s'auréolaient de lui, tous atteints par l'âge ou la mort, avaient disparu ou tout comme. Tout son cortège d'Hommes actifs et notoires s'ensevelissait dans l'oubli.

Il fallait trouver quelqu'un pour que lui, Aug. Chevalier, toute son œuvre et tout son entourage humain d'antan, ne s'efface pas du présent, que Demain soit encore Aug. Chevalier.

59 Des élèves ? Évidemment, il n'en avait personnellement formé aucun. Mais beaucoup s'étaient formés à son contact, d'eux-mêmes, donc les meilleurs, mais tous avaient cherché, hors son laboratoire, hors lui-même, une position sociale, tous une position de 
chercheurs ailleurs. Et je citais André Kopp, Didier Normand, Louis Hédin, Haudricourt, Roger de Vilmorin ${ }^{19}$, Jean Trochain, etc.... je lui en fis part.

Alors après m'avoir parlé, et de l'un et de l'autre, nettement heureux de ces résultats, il me demanda de bien vouloir prétendre à lui succéder et qu'il en serait très satisfait. J'acceptai.

61 Nous restâmes longtemps sans parler, moi revoyant en 1943 tout ce qui me reliait à lui depuis 1926.

En 1947, son désir, son besoin, était satisfait ${ }^{20}$.

En 1954, il s'en allait ${ }^{21}$.

Je garde d'Aug. Chevalier un souvenir ému. Il m'a pris sous son aile dès le début de moi en ne me le faisant pas penser, en ne s'exprimant pas. Je pense avoir, toutes modifications liées à l'évolution du monde moderne, continué Aug. Chevalier.

Ou bien me serais-je trompé ?

\section{BIBLIOGRAPHIE}

Bahuchet S. 2012 - Du JATBA-Revue d'ethnobiologie à la Revue d'ethnoécologie. Revue d'ethnoécologie [En ligne] 1, URL : http://ethnoecologie.revues.org/689

Bahuchet S., Blanc J., Hoare C., Juraver S., Kourdourli M. \& Pennec F. 2019 - Des hommes et des plantes. Revue d'ethnoécologie [En ligne] 16, URL : http://journals.openedition.org/ethnoecologie/ 5786

Bahuchet S. \& Lizet B. 2003 - L'ethnobotanique au Muséum national d'histoire naturelle. Les hommes, les idées, les structures. In : Lieutaghi P. \& Musset D. (Ed), Plantes, sociétés, savoirs, symboles. Matériaux pour une ethnobotanique européenne, «les cahiers de Salagon » n 8, Muséeconservatoire de Salagon et Les Alpes de lumière, Mane : 15-32.

Bonneuil C. 1996 - Auguste Chevalier, savant colonial; entre science et empire, entre botanique et agriculture. In : Petijean P. (Ed.), Les sciences coloniales. Figures et institutions (Les sciences hors d'occident au XX' siècle, vol. 2). Paris, Orstom : 15-36.

Chevalier A. 1929 - Nécrologie. Jean Dybowski (1855[sic]-1928). Revue de Botanique Appliquée et d'Agriculture Coloniale 9 (89) : 94-96.

Chevalier A. 1936 - Monographie de l'Arachide : l'arachide au Sénégal, 1936. Paris, Muséum national d'Histoire naturelle, Laboratoire d'Agronomie coloniale, 200 p.

Hoare C. 2012 - Le JATBA et ses ancêtres Revue d'ethnoécologie [En ligne], 1 | 2012, mis en ligne le 30 juin 2012, consulté le 10 décembre 2019. URL : http://journals.openedition.org/ethnoecologie/ 673

Jacques-Félix H. 1976 - Jean Trochain (1903-1976). JATBA 23 (1-3) : 57-58.

Leroy J.-F. 1957 - Auguste Chevalier (1873-1956). Bulletin de la Société Botanique de France, 104(sup. 1) : 65-77. DOI: 10.1080/00378941.1957.10835163 
Leroy J.-F. 1974 - Roland Portères : Professeur au Muséum National d'Histoire Naturelle (1906-1974). JATBA 21 (10-12) : 384-390.

Portères R. 1927 - La fermentation et la préparation du cacao. Revue de Botanique appliquée et d'Agriculture coloniale 7 (65) : 36-47.

Simon B. 2011 - Jean DYBOWSKI (Professeur) (1856-1928) Aux origines de l'agronomie tropicale en France. In : Serre J. (Ed.) Hommes et destins, T. XI, Afrique noire. Paris, l'Harmattan : 281-301.

\section{NOTES}

1. André Kopp agronome (1895-1947).

2. Émile Prudhomme (1871-1963), ingénieur agronome ; inspecteur de l'Agriculture à Madagascar (1896), directeur du Jardin colonial de Nogent-sur-Marne (1909), de l'École supérieur d'agriculture coloniale (1929), par la suite Institut d'agronomie coloniale, directeur de l'Institut national d'agronomie de la France d'outre-mer (1934).

3. Charles Chalot (1871- ?), Directeur du Jardin d'essai à Libreville (en 1897).

4. Jean Dybowsky (1856-1928).

5. Le Journal d'Agriculture Tropicale fut créé par Jean Vilbouchevitch au Jardin colonial de Nogent-sur-Marne (Hoare 2012).

6. La rivalité avec Dybowski est relatée par Bernard Simon : «Le Coffea Dybowskii n'a eu qu'une existence éphémère ; quant aux coffea congensis et excelsa, il ne nous a pas été possible de déterminer qui en était le véritable découvreur car Auguste Chevalier, qui prospecta la même région quelques années plus tard, s'en est attribué la paternité. Mais il détestait Dybowski qu'il considérait comme un amateur et un faux scientifique !". (Simon 2011). De plus, Chevalier écrit la notice nécrologique de Dybowski dans la Revue de botanique appliquée et d'agriculture coloniale qui confirme cette " acrimonie ". "Parmi les plantes les plus intéressantes ramenées en France à cette époque par la mission Dybowski, il faut citer le Coffea congensis Frœhner ou Caféier des rives de l'Oubangui et le $C$. Dybowskii Pierre, Caféier à grandes feuilles et petits fruits proche parent de la forme plus septentrionale que j'ai décrite sous le nom de $C$. excelsa. » [...] "À partir de 1910, Dybowski se confina dans son enseignement et il publia de loin en loin quelques notes sur des sujets d'actualité. Dans cet ordre d'idées il fut un vulgarisateur plus qu'un homme de sciences. Ses convictions ne reposaient pas toujours sur des faits scientifiques indiscutables. Elles étaient sincères, mais comme l'a dit M. H. Hitier, secrétaire perpétuel de l'Académie d'Agriculture: quelques-uns étaient tentés de le trouver parfois trop optimiste » (Chevalier 1929).

7. Jean Trochain (1903-1976), botaniste (Jacques-Félix 1976).

8. Philippe Van Tieghem (1839-1914), botaniste, directeur du laboratoire de botanique du Muséum à partir de 1879.

9. Buitenzorg (aujourd'hui Bogor) à Java, était la capitale des Indes néerlandaises ; un célèbre jardin botanique y avait été créé en 1817, à partir duquel furent diffusées de nombreuses plantes d'importance économique (thé, palmier à huile...).

10. Émile de Wildeman (1866-1947), botaniste belge. 
11. Paul Painlevé (1863-1933), mathématicien, membre du cartel des gauches, fut de nombreuses fois ministre et président du conseil ; il était ministre de la Guerre (de novembre 1925 à octobre 1929). La France s'engage dans la guerre du Rif en 1925-1926.

12. William Merlaud-Ponty (1866-1915), gouverneur général de l'AOF de 1908 à 1915 ; Albert Sarraut (1872-1962), gouverneur général de l'Indochine (1911-1914 puis 1917-1919), Ministre des colonies (1920-1924, puis 1932-1933).

13. Gaston Joseph (1884-1977), chef du cabinet du gouverneur de la Côte d'Ivoire (1917-1920), gouverneur du Cameroun (1924), directeur des affaires politiques au Ministère des colonies de 1925 à 1944.

14. Né en 1906, Roland Portères a alors 22 ans.

15. Paru auparavant dans la Revue de Botanique appliquée et d'Agriculture coloniale en 1933 et 1934 dans les fascicules 147-148, 156, 157 et 158.

16. Au Sénégal

17. Le Soudan français est devenu le Mali.

18. Théodore Monod (1902-2000), entré au Muséum en 1928, dirige l'IFAN de 1938 à 1965, bien qu'il soit élu professeur d'ichtyologie au Muséum en 1946.

19. Didier Normand agronome et anatomiste du bois (1908-2008), Louis Hédin agronome et botaniste (1904-1982), André-Georges Haudricourt agronome, ethnobotaniste et linguiste (1911-1996), Roger de Vilmorin horticulteur et généticien (1905-1980).

20. Roland Portères a été élu professeur d'agronomie tropicale au Muséum en 1948 ; il dirigera le laboratoire devenu ethnobotanique jusqu'à son décès en 1974.

21. Ici Portères fait une erreur sur la date, Auguste Chevalier est mort en 1956. 